Supporting Information for

\title{
Constructing High-Loading Single-Atom/Cluster Catalysts via an Electrochemical Potential Window Strategy
}

\author{
Jin-Cheng Liu ${ }^{1}$, Hai Xiao ${ }^{*}, 1$, and Jun $\mathrm{Li}^{*}, 1,2$ \\ ${ }^{1}$ Department of Chemistry, Tsinghua University, Beijing 100084, China \\ 2 Department of Chemistry, Southern University of Science and Technology, Shenzhen 518055, \\ China \\ E-mails: haixiao@tsinghua.edu.cn; junli@tsinghua.edu.cn
}



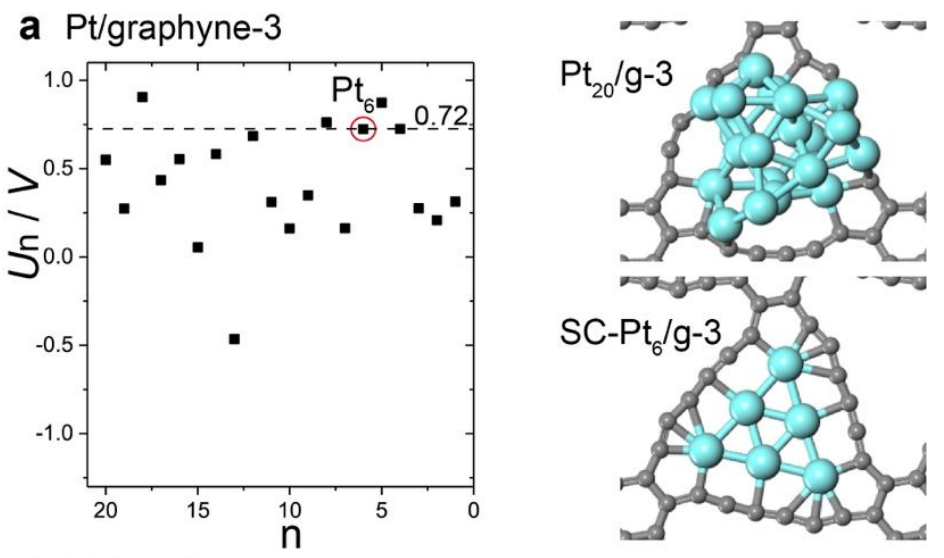

b Pd/graphyne-3
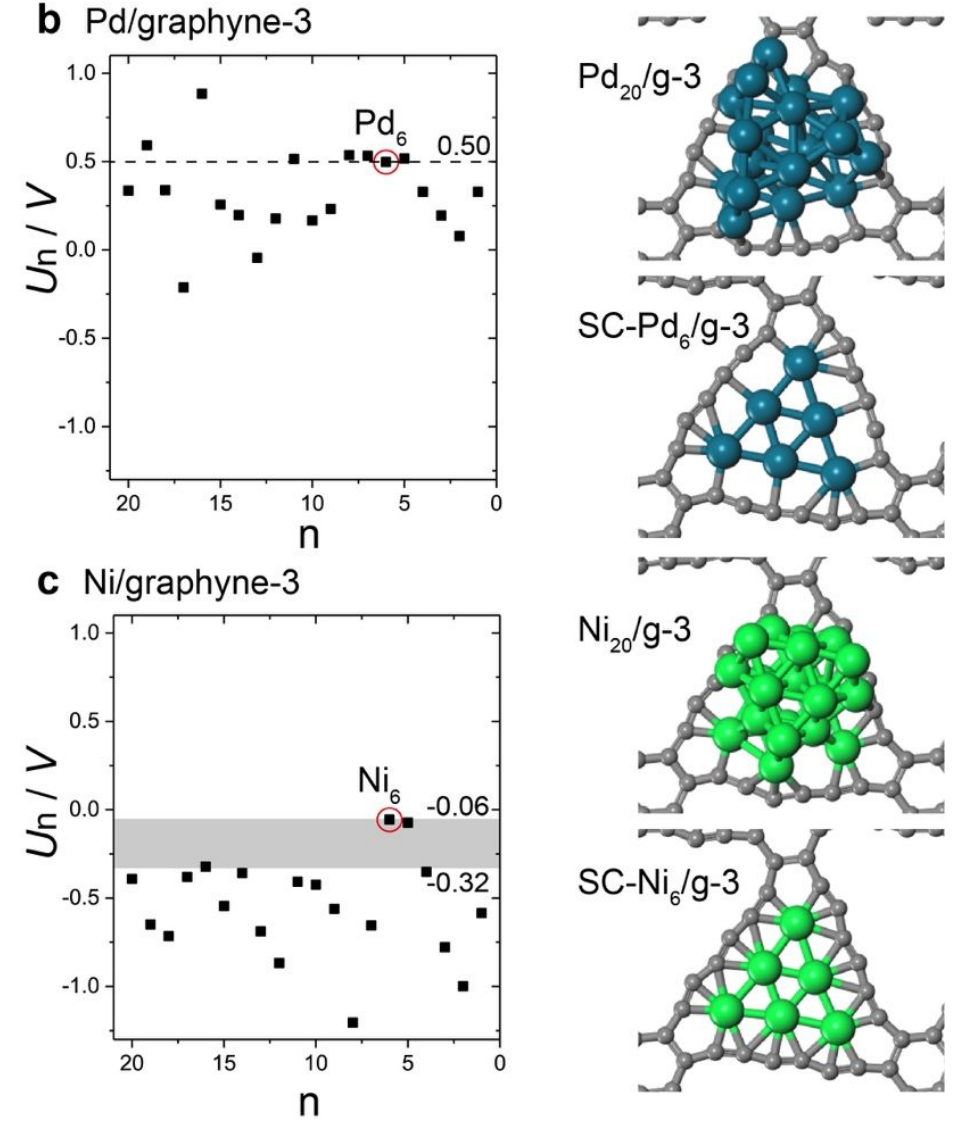

Figure S1. The threshold leaching potentials $U_{n}$ (vs SHE) for $M_{n} /$ graphyne-3 (g-3), with $M=P t$ (a), Pd (b), Ni (c). The grey area marks the potential window, within which all forms of metals (including NPs) are depleted, except for the $\mathrm{M}_{6} \mathrm{SC}$ at the hole of $\mathrm{g}-3$. The optimized configurations of $\mathrm{M}_{20} / \mathrm{g}-3$ and $\mathrm{SC}-\mathrm{M}_{6} / \mathrm{g}-3$ are shown alongside. The color code for elements is: $\mathrm{Pt}$ (cyan); $\mathrm{Pd}$ (dark green); Ni (light green); C (gray). 


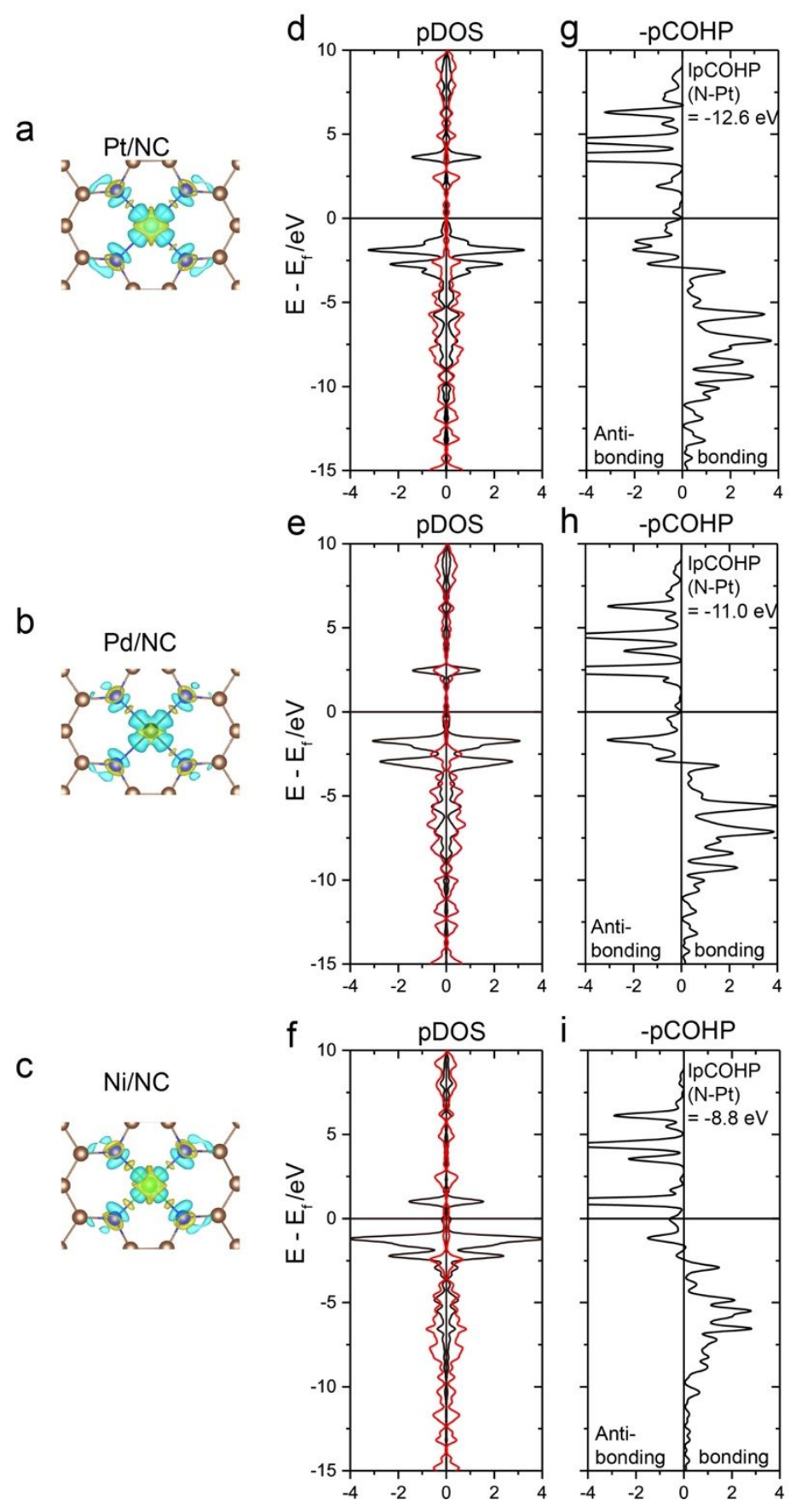

Figure S2. The calculated charge density differences for (a) Pt, (b) Pd, (c) Ni SAs on NC, where the yellow and cyan contours represent electron density increase and depletion, respectively. The projected densities of states (pDOS) of (d) Pt, or (e) Pd, or (f) Ni atom (black curve) and adjacent $\mathrm{N}$ (red curve). The summed projected crystal orbital Hamilton population ( $\mathrm{pCOHP}$ ) for the four (g) $\mathrm{N}-\mathrm{Pt}$, or (h) N-Pd, or (i) N-Ni bonds and its integrated value (IpCOHP). 

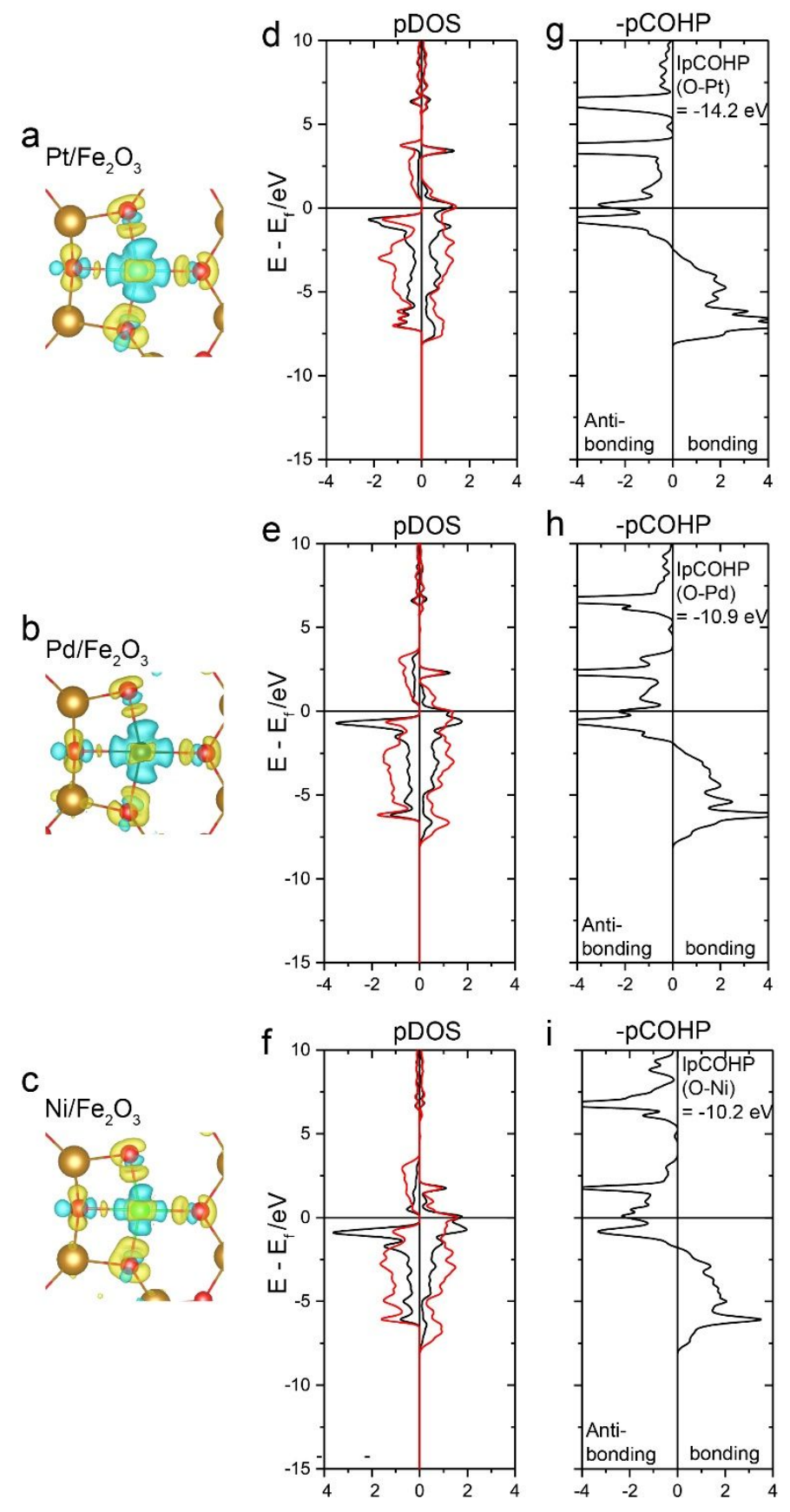

Figure S3. The calculated charge density differences for (a) Pt, (b) Pd, (c) Ni SAs on $\mathrm{Fe}_{2} \mathrm{O}_{3}$, where the yellow and cyan contours represent electron density increase and depletion, respectively. The projected densities of states (pDOS) of (d) Pt, or (e) Pd, or (f) Ni atom (black curve) and adjacent $O$ (red curve). The summed projected crystal orbital Hamilton population (pCOHP) for the four (g) O-Pt, or (h) O-Pd, or (i) O-Ni bonds and its integrated value (IpCOHP). 


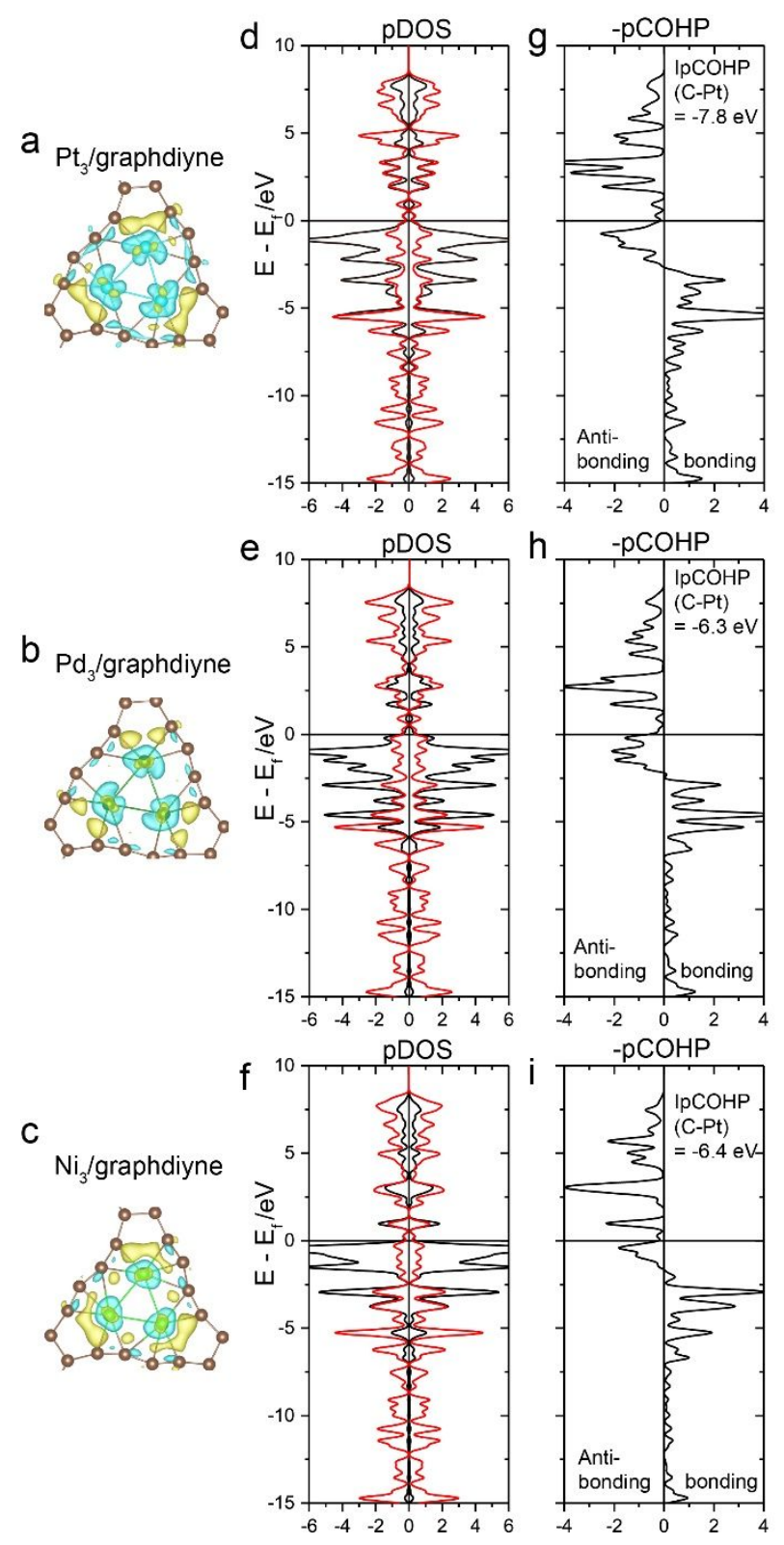

Figure S4. The calculated charge density differences for (a) $\mathrm{Pt}_{3}$, (b) $\mathrm{Pd}_{3}$, (c) $\mathrm{Ni}_{3}$ SCs on graphdiyne, where the yellow and cyan contours represent electron density increase and depletion, respectively. The projected densities of states (pDOS) of (d) Pt, or (e) Pd, or (f) Ni atom (black curve) and adjacent $C$ (red curve). The summed projected crystal orbital Hamilton population ( $\mathrm{pCOHP}$ ) for the five (g) C-Pt, or (h) C-Pd, or (i) C-Ni bonds and its integrated value (IpCOHP). 

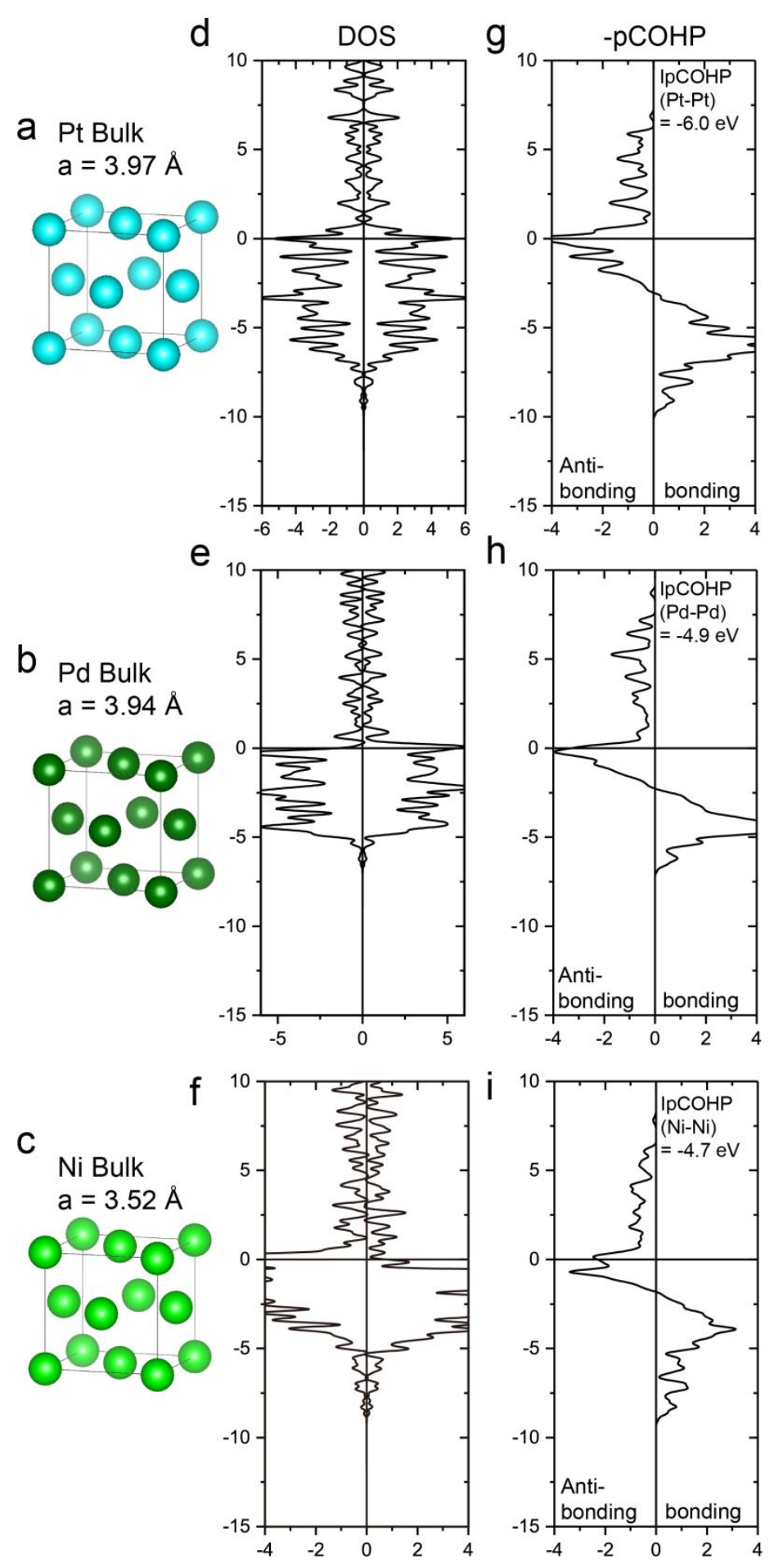

Figure S5. The optimized bulk (a) Pt, (b) Pd, and (c) Ni. The densities of states (DOS) of (d) Pt, (e) Pd, and (f) Ni bulk metals. The summed projected crystal orbital Hamilton population (pCOHP) for twelve (g) Pt-Pt, or (h) Pd-Pd, or (i) Ni-Ni bonds and its integrated value (IpCOHP). 
Table S1. The Bader, Mulliken and Löwdin atomic charges in $\mathrm{Pt}_{1} / \mathrm{NC}, \mathrm{Pd}_{1} / \mathrm{NC}, \mathrm{Ni}_{1} / \mathrm{NC}, \mathrm{Pt}_{1} / \mathrm{Fe}_{2} \mathrm{O}_{3}$, $\mathrm{Pd}_{1} / \mathrm{Fe}_{2} \mathrm{O}_{3}, \mathrm{Ni}_{1} / \mathrm{Fe}_{2} \mathrm{O}_{3}, \mathrm{Pt}_{3} /$ graphdiyne, $\mathrm{Pd}_{3} /$ graphdiyne, $\mathrm{Ni}_{3} /$ graphdiyne.

\begin{tabular}{|c|c|c|c|c|c|c|}
\hline & \multicolumn{2}{|c|}{ Bader charge } & \multicolumn{2}{c|}{ Mulliken charge } & \multicolumn{2}{c|}{ Löwdin charge } \\
\hline & metal & $\mathrm{N}$ & metal & $\mathrm{N}$ & metal & $\mathrm{N}$ \\
\hline $\mathrm{Pt}_{1} / \mathrm{CN}$ & 1.00 & -2.56 & 1.11 & -0.59 & 0.87 & -0.38 \\
\hline $\mathrm{Pd}_{1} / \mathrm{CN}$ & 0.92 & -2.57 & 0.86 & -0.53 & 0.64 & -0.32 \\
\hline $\mathrm{Ni}_{1} / \mathrm{CN}$ & 1.00 & -2.55 & 1.10 & -0.58 & 0.88 & -0.38 \\
\hline & Metal & $\mathrm{O}$ & metal & $\mathrm{O}$ & metal & $\mathrm{O}$ \\
\hline $\mathrm{Pt}_{1} / \mathrm{Fe}_{2} \mathrm{O}_{3}$ & 1.41 & -0.93 & 1.47 & -0.88 & 1.35 & -0.81 \\
\hline $\mathrm{Pd}_{1} / \mathrm{Fe}_{2} \mathrm{O}_{3}$ & 1.18 & -0.89 & 1.13 & -0.84 & 1.00 & -0.75 \\
\hline $\mathrm{Ni}_{1} / \mathrm{Fe}_{2} \mathrm{O}_{3}$ & 1.32 & -0.96 & 1.36 & -0.90 & 1.21 & -0.82 \\
\hline & Metal & $\mathrm{C}$ & metal & $\mathrm{C}$ & metal & $\mathrm{C}$ \\
\hline $\mathrm{Pt}_{3} / \mathrm{graphdiyne}$ & 0.31 & -0.16 & 0.82 & -0.17 & 0.67 & -0.15 \\
\hline $\mathrm{Pd}_{3} / \mathrm{graphdiyne}$ & 0.32 & -0.16 & 0.60 & -0.13 & 0.44 & -0.10 \\
\hline $\mathrm{Ni}_{3} / \mathrm{graphdiyne}$ & 0.51 & -0.17 & 0.92 & -0.20 & 0.71 & -0.16 \\
\hline
\end{tabular}




\section{Benchmark of computational scheme on predicting the leaching potential}

To benchmark our computational method directly against the experiment, we chose to calculate the electrochemical potential for leaching a single Pt atom from the monolayer step on $\mathrm{Pt}(111)$ surface, for which there are experimental results available for direct comparison. ${ }^{1}$ The experimental cyclic voltammograms show that the onset potential for etching step atoms on $\mathrm{Pt}(111)$ is $0.65 \mathrm{~V}$ with a $\mathrm{Pt}$ concentration of $0.926 \mathrm{ppb}\left(0.926 \mu \mathrm{g} / \mathrm{kg}, 4.74 \times 10^{-12} \mathrm{~mol} / \mathrm{L}\right)$.

We employed a $\mathrm{Pt}(322)-\mathrm{p}(1 \times 3)$ four-layer surface slab to model the monolayer step on $\mathrm{Pt}(111)$.

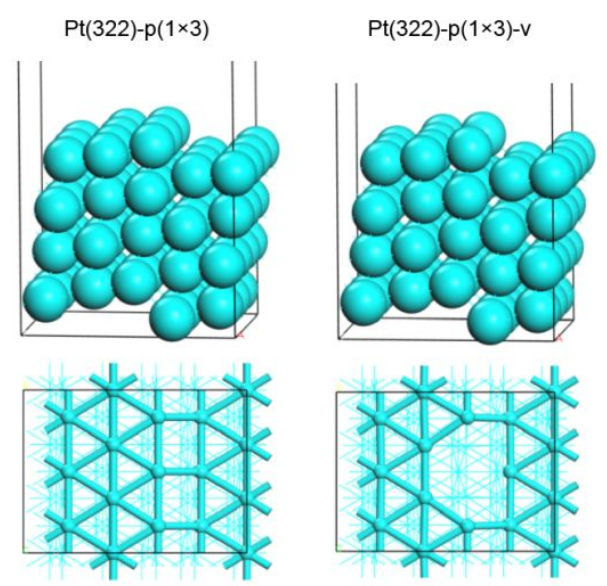

Figure S6. $\mathrm{Pt}(322)-\mathrm{p}(1 \times 3)$ four-layer surface slab before and after leaching one step $\mathrm{Pt}$ atom.

With our computational methodology, we predicted a threshold potential $U$ of $0.623 \mathrm{~V}$ for leaching a Pt step atom on $\mathrm{Pt}(111)$ with $\left[\mathrm{Pt}^{2+}\right]=4.74 \times 10^{-12} \mathrm{~mol} / \mathrm{L}$ (this effect is included by the Nernst equation).

$\mathrm{Pt}^{2+}(\mathrm{aq})+2 \mathrm{e}^{-} \rightleftharpoons \mathrm{Pt}(\mathrm{s}), \mathrm{U}_{0}\left(\left[\mathrm{Pt}^{2+}\right]=1 \mathrm{~mol} / \mathrm{L}\right)=+1.188 \mathrm{~V}$.

Based on Nernst equation, $U=U_{0}-R T / z F \times \log \left(\left[\mathrm{Pt}^{2+}\right]\right)$

When $\left[\mathrm{Pt}^{2+}\right]=4.74 \times 10^{-12} \mathrm{~mol} / \mathrm{L}, U_{0}\left(\left[\mathrm{Pt}^{2+}\right]=4.74 \times 10^{-12} \mathrm{~mol} / \mathrm{L}\right)=+0.853 \mathrm{~V}$,

The energies obtained from JDFTx are shown below,

\begin{tabular}{|l|l|l|l|l|}
\hline & \multicolumn{4}{|c|}{ Free energy /a.u. } \\
\hline & $\operatorname{Pt}(322)-\mathrm{p}(1 \times 3)$ & $\mathrm{Pt}(322)-\mathrm{p}(1 \times 3)-\mathrm{V}$ & $\mathrm{Pt}($ bulk $)$ & $\mathrm{Pt}^{2+}(0.926 \mathrm{ppb})$ \\
\hline$U=0 \mathrm{~V}$ & -5178.120064 & -5091.800211 & -88.928465 & -86.255098 \\
\cline { 1 - 3 } & & & & \\
\cline { 1 - 3 } & -5107.568954 & -5022.427197 & & -85.137914 \\
\hline
\end{tabular}


$U_{\mathrm{n}}=\left(G_{0, \mathrm{n}}+2 \mathrm{e} U_{0}-G_{0, \mathrm{n}-1}-G(M(\mathrm{~s}))\right) /\left(\mathrm{k}_{\mathrm{n}-1}-\mathrm{k}_{\mathrm{n}}+2 e\right)$.

$U_{\mathrm{n}}=0.623 \mathrm{~V}$.

This result is close to the experimentally reported onset potential of $0.65 \mathrm{~V} .{ }^{1}$ Thus, our method is reliable for predicting the threshold potential for electrochemical leaching. It is worth emphasizing that the prediction of electrochemical potentials is very likely to have some systematic errors because of the approximation in DFT (GGA level of approximation used here) and computational models etc, but these systematic errors would only shift the positions of predicted electrochemical potential windows and would not affect the qualitative conclusions here. 


\section{Comparison of oxidation threshold potentials from simulated annealing and optimization}

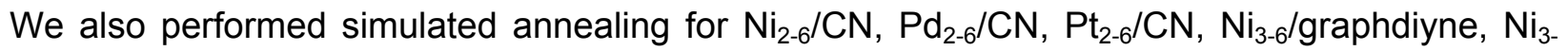
${ }_{6} /$ graphdiyne, $\mathrm{Ni}_{3-6} /$ graphdiyne from $1000 \mathrm{~K}$ to $100 \mathrm{~K}$, followed by structure optimization. And then we calculated the threshold potentials $U$ based on these structures. The results show that there is still a sharp jump of energy from small clusters to the SA/SC form and thus the electrochemical potential window, which is in complete consistence with our results from geometry optimization. We found that the results from simulated annealing differ little from the results from geometry optimization.
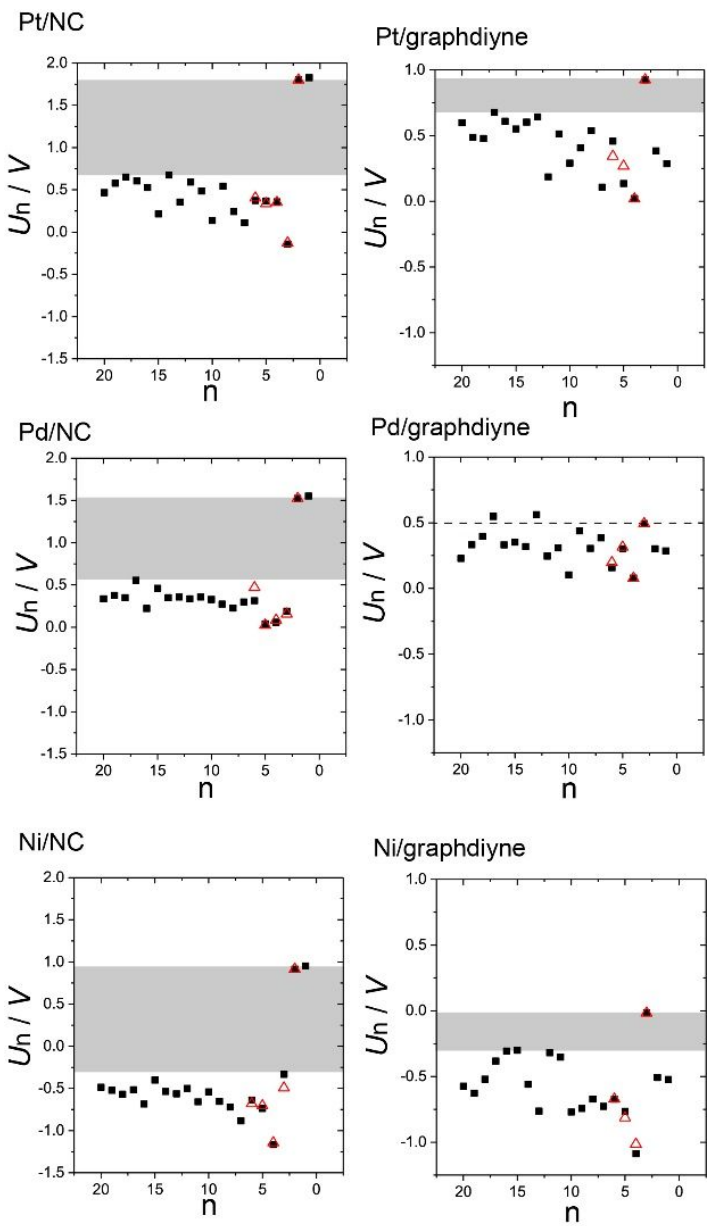

Figure S7. The threshold leaching potentials $U_{n}$ (vs SHE) for $M_{n} / N C$ and $M_{n} /$ graphdiyne, with $M$ $=\mathrm{Pt}, \mathrm{Pd}, \mathrm{Ni}$. The red points are calculated from simulated annealing structures, and black points are from optimization. 
1. Komanicky, V.; Chang, K. C.; Menzel, A.; Markovic, N. M.; You, H.; Wang, X.; Myers, D., Stability and Dissolution of Platinum Surfaces in Perchloric Acid. J. Electrochem. Soc. 2006, 153 (10), B446-B451. 